\title{
SUBTENONS INFECTION BY DIROFILARIA REPENS
}

\author{
*P Sathyan, P Manikandan, M Bhaskar, S Padma, G Singh, B Appalaraju
}

\begin{abstract}
Zoonotic filariasis caused by Dirofilaria repens, a parasite of carnivores occurs mainly in countries surrounding Mediterranean region. The infection occurs in and around eye among animal handlers through vector transmission. We are reporting a case of human Dirofilariasis affecting subtenons region in a 63 year-old woman from Coimbatore, South India. The purpose of this article is to review the clinical course and management of ocular Dirofilariasis.
\end{abstract}

Key words: dirofilariasis, dirofilaria repens, subcutaneous

Ocular zoonotic infections by filarial worms are not uncommon. Most of them are caused by the genus Dirofilaria. ${ }^{1}$ The original name of $D$. conjunctivae for worms causing subcutaneous Dirofilariasis suggests the preferential localization of these worms in and around the eye. Though these infections are widely reported from USA, Europe and south East Asia, not many cases occur in India. Few case reports involving Dirofilaria repens infecting ocular adnexa are the only available literature..$^{1,2}$

Here we report a case of subtenons Dirofilariasis in a 63 year-old woman from Coimbatore, south India. The purpose of this article is to review the clinical features and course of management, as subtenons involvement in ocular Dirofilariasis is relatively uncommon.

\section{Case Report}

A 63 year-old lady reported to Aravind Eye Hospital, Coimbatore, South India with complaints of pain over left supraorbital region, vomiting and redness on nasal side of left eye for one week. She had consulted a local doctor and was treated with topical antibiotics and steroids. After a brief period of relief the symptoms recurred with an added swelling and pain in the left eye. There was no history of injury, allergy or any previous lesions in the left eye. There was no history of travel anywhere from her place in past one year. There was no history of any major systemic illness and she was not a diabetic or hypertensive.

Ocular examination revealed an undulating vermiform mass by a live worm on the nasal quadrant of left eye under bulbar conjunctiva with localized congestion (Fig. 1a). Rest of the ocular examination was with in normal limits and her

*Corresponding author (e-mail: <sathyan.p@cbe.aravind.org>)

Aravind Eye Hospital and Post Graduate Institute of Ophthalmology (PS, PM, MB,SP,GS) Coimbatore-641 014, Tamilnadu, India; and PSG institute Medical Sciences (BA), Coimbatore-641 014, Tamilnadu, India

Received: 05-01-05

Accepted: 15-02-05 visual acuity was $6 / 6$ in both eyes. The haematological and biochemical test results were normal and no microfilaremia was seen in blood smears. The worm was removed intact surgically without damaging its structures (Fig. 1b), preserved in $10 \%$ formaldehyde and sent for identification.

\section{Parasitological findings}

The worm was thin, cylindrical and measured about 9.5 $\mathrm{cm}$ in length with a maximum diameter of $536 \mu \mathrm{m}$. The anterior end (Fig. 2a) was bluntly rounded and of greater diameter than the posterior end (Fig. 2b). The genital pore was located $1.3 \mathrm{~mm}$ from the anterior end. The cuticle of the worm was thick, had marked longitudinal ridges with transverse striations. The uterus was didelphic and opisthodelphic, packed with immature eggs. The anus opened almost terminally. The tail was $100 \mu \mathrm{m}$ long and had obtuse tip. The

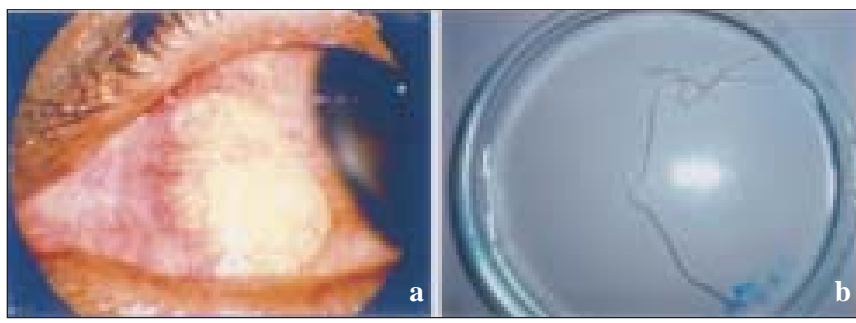

Figure 1: Clinical appearance of adult worm under subtenon's space (a) and full view of the adult worm (b)

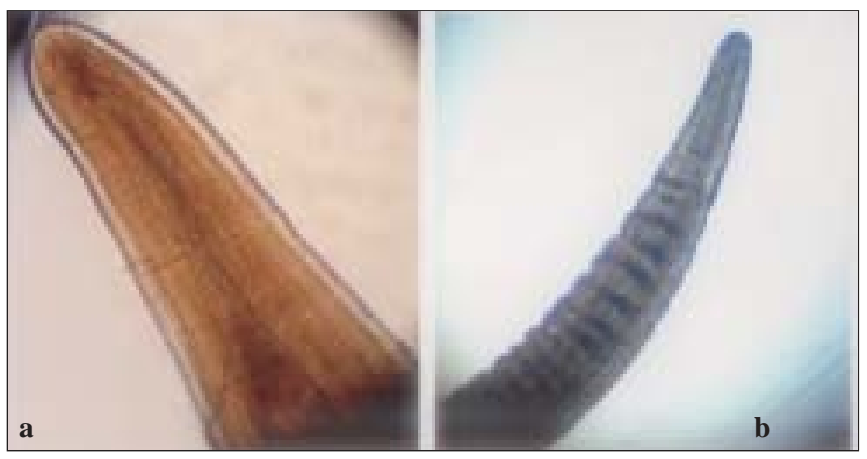

Figure 2: Photomicrograph of anterior end of worm (a) and surface of cuticle and posterior end (b). 
worm was unfertilized or immature. Based on the size, cuticular and internal morphologic features the worm was identified as an unfertilized female $D$. repens.

\section{Discussion}

Dirofilariasis is often reported from European countries surrounding the Mediterranean particularly from Italy. ${ }^{1}$ The other countries that have reported these infections are France, Greece and Israel. ${ }^{1}$ In India, subcutaneous Dirofilariasis is rare, only few cases having been reported from Karnataka and Kerala. ${ }^{3-5}$ Ocular Dirofilariasis is a form of subcutaneous Dirofilariasis caused by D. repens. D. repens is a natural parasite of carnivorous animals, primarily dogs, foxes and cats. $^{2,3}$ The other causative organism $D$. tenuis, reported from USA, is a parasite of raccoons. ${ }^{2}$ Some authors consider that $D$. repens is synonymous with $D$. conjunctivae as well as $D$. tenuis. ${ }^{3}$ The infection is transmitted to humans by insect vector like mosquito. ${ }^{6}$ The percentage of parasitaemia from $D$. repens in dogs varies from 12 to $37 \%{ }^{6}$

Zoonotic filariasis occurs among those individuals who handle cats and dogs (either as pets, as in our case or as in professions like veterinary doctors). Subcutaneous dirofilariasis normally affects the eyelids and periorbital region and at rare occasion they may occur subconjunctivally. ${ }^{2}$

The diagnosis is confirmed by studying the morphology after their removal. Worms belonging to the genus Dirofilaria are identified by their thick laminated cuticle, broad lateral ends and large muscle cells. The length of female may vary from 8 to $13 \mathrm{~cm}$ and males from 4 to $4.8 \mathrm{~cm}^{2}$ Though the worms with external longitudinal cuticular ridges ${ }^{2,3}$ are taken as $D$. repens there are other Dirofilaria, which may show these ridges. Exact identification of species may be possible only after studying the fully matured worm. However $D$. immitis can be differentiated from $D$. repens by absence of ridges. $D$. immitis also causes microfilaremia in human and requires antihelminthic drugs, where as microfilaremia is absent in human cases of infection caused by $D$. repens and there is no documentation of use of antihelminthic drugs in any literature.

Based on the size of the worm, it is likely that the patient had been infected at least 6 months, probably much longer, and had not exhibited symptoms until the worm entered the conjunctiva of the eye. The treatment in cases with zoonotic
Dirofilariasis is surgical removal of the worm. Symptoms resolved quickly in our case following surgical removal. We have not encountered any difficulty in surgical removal of the worm, because the worm had only restricted movement, otherwise we can use cryoprobe for immobilizing the worm as described by Gendelman et al. ${ }^{7}$ No further treatment was necessitated in our case because only a single worm was present.

Ruiz-Moreno et $a l^{1}$ have demonstrated high titre of antibodies and Aravanitis et $a l^{6}$ could not detect any antibody raise following infection. In our country owing to the low prevalence rate no standard antibody detecting tests are available for $D$. repens infection, which can be used as another confirmatory test. Human cases of Dirofilariasis are most probably underreported because many of them remain undiagnosed or unpublished. ${ }^{3}$ Increased awareness of this infection among ophthalmologists and development of antibody detecting system may improve patient care and also increase the prevalence rate.

\section{References}

1. Ruiz-Moreno JM, Bornay-Llinares FJ, Maza GP, Medrano M, Simon F, Eberhard ML. Subconjunctival Infection with Dirofilaria repens: Serological Confirmation of Cure Following Surgery. Arch Ophthalmol 1998;116:1370-2.

2. Font RL, Neafie RC, Perry HD. Subcutaneous Dirofilariasis of the Eyelid and Ocular Adnexa: Report of six cases. Arch Ophthalmol 1980;98:1079-82.

3. Gautam V, Rustagi M, Singh S, Arora DR. Subconjunctival infection with Dirofilaria repens. Jpn J Infect Dis 2002;55:47-8.

4. Sekhar HS, Srinivasa H, Batru R, Mathai E, Shariff S, Macaden RS. Human ocular Dirofilariasis in Kerala South India. Indian J Pathol Micorbiol 2000;43:77-9.

5. Nadgir S, Tallur SS, Mangoli V, Halesh LH, Krishna BV. Subconjunctival Dirofilariasis in India. South Asian J Trop Med Public Health 2001;32:244-6.

6. Aravanitis PG, Vakalis NC, Damanakis AG, Theodossiadis GP. Ophthalmic Dirofilariasis. Am J Ophthalmol 1997;123:689-91.

7. Gendelman D, Blumberg R, Sadun A. Ocular Loa Loa with Cryoprobe Extraction of Subconjunctival Worm. Ophthalmology 1984;91:300-3. 